\title{
The Application of Wavelet-Based Least Mean Square Algorithm in Adaptive Beamforming
}

\author{
Shun-Hsyung Chang \\ Associate Professor, Department of Electrical Engineering, National Taiwan Ocean University, Keelung, Taiwan, R.O.C., \\ b0091@ntou66.ntou.edu.tw \\ Chin-Chang Chang \\ Graduate Student, Department of Electrical Engineering, National Taiwan Ocean University, Keelung, Taiwan, R.O.C.
}

Follow this and additional works at: https://jmstt.ntou.edu.tw/journal

Part of the Electrical and Computer Engineering Commons

\section{Recommended Citation}

Chang, Shun-Hsyung and Chang, Chin-Chang (1997) "The Application of Wavelet-Based Least Mean Square Algorithm in Adaptive Beamforming," Journal of Marine Science and Technology. Vol. 5: Iss. 1, Article 2.

DOI: $10.51400 / 2709-6998.2533$

Available at: https://jmstt.ntou.edu.tw/journal/vol5/iss1/2

This Research Article is brought to you for free and open access by Journal of Marine Science and Technology. It has been accepted for inclusion in Journal of Marine Science and Technology by an authorized editor of Journal of Marine Science and Technology. 


\section{The Application of Wavelet-Based Least Mean Square Algorithm in Adaptive Beamforming}

\section{Acknowledgements}

This work was supported by National Science Council, Taiwan, R.O.C. under the program NSC 84-2213-E-019-015. The authors also wish to thank the reviewers for numerous helpful suggestions. 


\title{
THE APPLICATION OF WAVELET-BASED LEAST MEAN SQUARE ALGORITHM IN ADAPTIVE BEAMFORMING
}

\author{
Shun-Hsyung Chang,* and Chin-Chang Chang**
}

Keywords: Wavelet transform, Adaptive Beamforming, LMS algorithm, SONAR.

\section{ABSTRACT}

Wavelet transform is one of methods that utilizes the dilation and contraction of prototype wavelet basis function to form a set of fully flexible windows in the time-frequency plane. It exhibits a good effect in both the analysis of high and low frequency components of signal. By applying such a transform method to traditional least-mean-square algorithm (LMS), we can obtain an orthogonally transformed signal from a time-domain input signal after imposing wavelet transform on it. In addition, we can improve the convergence speed of learning curve by passing the transformed signal through weight which is adapted with LMS. The improved method is called the wavelet-based least-mean-square algorithm (WLMS).

In this paper, we impose the WLMS on the traditional adaptive beamformer, the one that used the time-domain input signal with LMS algorithm (TLMS) to adjust the system weights. Instead of TLMS, we adapt WLMS here to improve the convergence speed of adaptive beamformer. Besides, we further demonstrate theoretical analysis and computer simulations to verify the performance of our transform method.

\section{INTRODUCTION}

In recent years, the theory and technology of adaptive beamforming have been applied extensively in many different areas such as RADAR, SONAR, communication systems, and geophysical exploration [1-3]. The applications of wavelet subband decomposition in adaptive arrays [4] also become very popular currently. More interestedly, the subbanding, where the wavelet transform is implemented prior to

Paper Received February, 1997. Revised April, 1997. Accepted April, 1997. Author for Correspondence: Shun-Hsyung Chang.

*Associate Professor, Department of Electrical Engineering, National Taiwan Ocean University, Keelung, Taiwan, R.O.C. Email: b0091@ntou66.ntou.edu.tw

**Graduate Student, Department of Electrical Engineering, National Taiwan Ocean University, Keelung, Taiwan, R.O.C. adaptive processing and tap delay filtering, is applied on hot clutter mitigation [5]. The convergence of weights reacts in very slow speed for traditional adaptive beamformer that used time-domain least mean square algorithm (TLMS), in which the eigenspread of autocorrelation matrix for input signal is distributed too spread or when it is a nonstationary signal [6-8]. The signal processing experts have attempted looking for remedies to overcome this problem. They aim at nonstationary signals which are frequently come across in engineering applications. Several time-frequency relevant analysis tools have been recommended such as the brand new wavelet transform in signal analysis. The wavelet transform utilizes the scaling and translation of mother wavelet function to form a set of fully flexible windows in time-frequency plane [9]. And as such, the data extraction from signals is expected to be equal in both the analysis of high and low frequencies.

This paper will combine the wavelet transform and the least mean square algorithm in order to improve the convergence speed of system weights, where we've named it as wavelet-based least mean square algorithm (WLMS). To reach the cancellation of jammer in rapid manner, we apply the WLMS algorithm on adaptive beamformer. We make use of the generalized sidelobe canceller (GSC) which proposed by Griffiths and Jim [10] that based on the linearly constrained minimum variance (LCMV) proposed by Frost [11]. Further, Frost implemented the LCMV's softly constrained condition by using the hardware techniques to reduce the complexity of array system. The problem of complexity reduction has also emerged as a hot issue in satellite communications [12]. In this paper, we are using such kind of adaptive beamformer framework in conjunction with the WLMS algorithm in order to improve its capability. 
In order to increase the convergence speed of the least mean square algorithm in time domain, we transform the time-domain signal into its orthogonal transform-domain contents. Subsequently, let the orthogonal transform signal become input signal of the least mean square algorithm. As such, faster convergence performance can be achieved. Since the utilization of Fast Fourier Transform, an orthogonal transform, can greatly reduce the computational complexity, it is being used widely by some researchers.

The Fourier transform's utility lies in its ability to analyze a stationary signal for the weights summation of its different sinusoidal contents. It transforms the time-domain signals into their orthogonal frequency-domain contents. However, signal occurred in most of engineering applications are nonstationary, because they are generated by systems whose statistical properties of parameters vary with time. Even so, Fourier transform would encountered its difficulty when analyzing time-varying nonstationary signals into their appropriate sinusoidal contents. Several time-frequency related analysis have been suggested successively to remedy the problem that come across by Fourier transform analysis. Wavelet transform constructed from mother wavelet function, in which persists good resolution, emerges among the others to become one of the best tools. The wavelet transform maintains a very good resolution in timefrequency domain. It not only preserves the benefits of Fourier transform in the processing of stationary signals, but also successfully utilizes the concept of mult-iresolution analysis to decompose timevarying nonstationary signals into their different orthogonal subspaces [7,13]. As demonstrated in Fig. 1, with the application of wavelet transform on the least mean square algorithm, we are led to a full understanding about the wavelet domain signal $v_{m n}(k)$, where it is obtained from time-domain input signal $x(k)$ by using the mother wavelet function transformation:

$$
v_{m n}(k)=\sum_{l} \psi_{m n}(k-l) x(l) .
$$

The quantity $\psi_{m n}$ is an orthogonal wavelet basis function, and $m, n$ are scaling and translation parameters respectively. Express the wavelet-domain signal vector $\mathbf{v}(k)$ of (1) in the matrix form:

$$
\mathbf{v}(k)=\Psi \mathbf{x}(k),
$$

where

$$
\mathbf{x}(k)=[\ldots, x(k+1), x(k), x(k-1), \ldots]^{T} .
$$

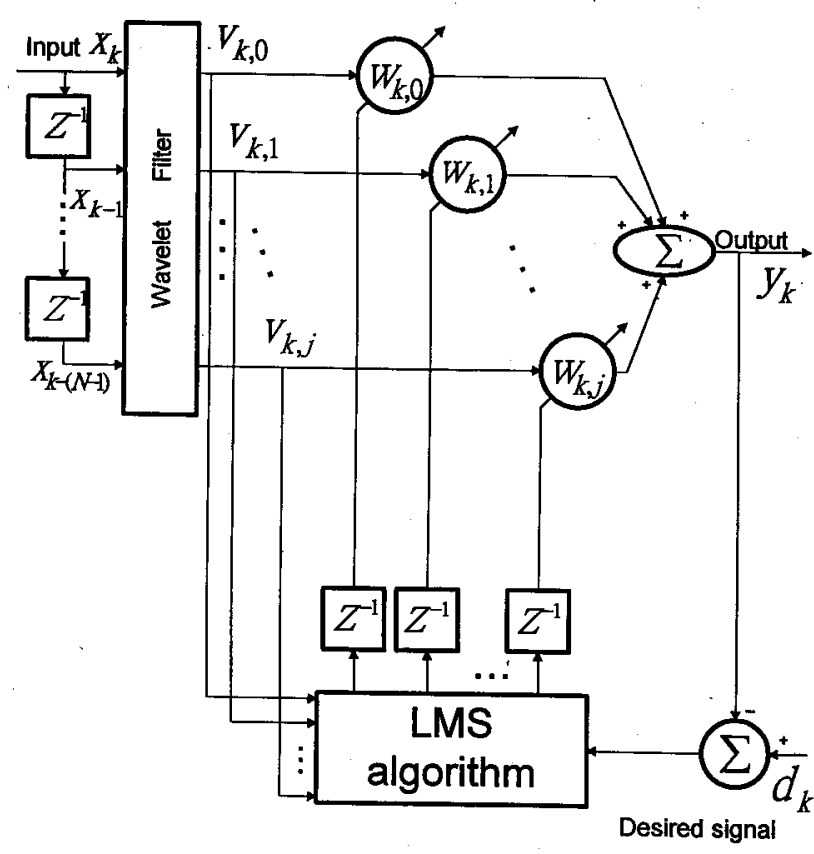

Fig. 1. Block diagram for the application of wavelet transform upon the least mean square.

The wavelet transform matrix is denoted as $\Psi$, while its row vector is $\Psi_{m n}$, at which

$$
\Psi_{\mathrm{mn}}=\left[\ldots, \psi_{m n}(k-1), \psi_{m n}(k), \psi_{m n}(k+1), \ldots\right]
$$

The system output signal $y(k)$ can be obtained from $\mathbf{V}(k)$ by using the weights $\mathbf{W}(k)$, as shown below:

$$
y(k)=\mathbf{W}(k) \mathbf{V}(k),
$$

where

$$
\mathbf{W}(k)=[\ldots, w(k+1), w(k), w(k-1), \ldots] .
$$

Follow the derivation of Wiener-Hopf equation [14, 15] to obtain the optimum weights for wavelet domain,

$$
\mathbf{w}_{o p t}^{\mathbf{W}}=\mathbf{R}_{v}^{-1} \mathbf{p}_{v},
$$

where $\mathbf{R}_{v}=E\left[\mathbf{v}(k)\left\{\mathbf{v}(k)^{*}\right]\right.$ is the autocorrelation matrix for input signal in wavelet-domain: $\mathbf{p}_{v}=$ $E[\overline{d(k)} \mathbf{v}(k)]$ signifies the cross-correlation vector between desired output signal and input signal vector within wavelet-domain.

With the substitution of $\mathbf{v}(k)$ from Eq. (2) into $\mathbf{R}_{v}, \mathbf{p}_{v}$, we have

$$
\begin{aligned}
\mathbf{R}_{v} & =\Psi E\left[\mathbf{x}(k) \mathbf{x}(k)^{*}\right] \Psi * \\
& =\Psi \mathbf{R}_{x} \Psi^{*}
\end{aligned}
$$




$$
\begin{aligned}
\mathbf{P}_{v} & =\Psi E[\overline{d(k)} \mathbf{x}(k)] \\
& =\Psi \mathbf{p}_{x} .
\end{aligned}
$$

Hence, the minimum mean square error in wavelet domain can further be derived as follow $[6,16]$,

$$
\begin{aligned}
\epsilon_{\min }^{W} & =E[d(k) \overline{d(k)}]-\mathbf{p}_{v} * \mathbf{R}_{v}^{-1} \mathbf{p}_{v} \\
& =E[d(k) \overline{d(k)}] \\
& -\left(\mathbf{p}_{x} * \Psi *\right)\left((\Psi *)^{-1} \mathbf{R}_{x}^{-1} \Psi^{-1}\right)\left(\Psi_{\mathbf{p}_{x}}\right) \\
& =E[d(k) \overline{d(k)}]-\mathbf{p}_{x}{ }^{*} \mathbf{R}_{x}^{-1} \mathbf{p}_{x} \\
& =\epsilon_{\min } .
\end{aligned}
$$

The above derivation reveals the minimum mean square error of wavelet-domain is equal to that of time domain. To adjust the weights, $\mathbf{W}$, we apply the least mean-square algorithm in the form of

$$
\mathbf{w}(k+1)=\mathbf{w}(k)+\mu \mathbf{v}(k) \overline{e(k)} .
$$

The range of convergence factor $\mu$ is constrained by

$$
0<\mu<\frac{2}{\operatorname{tr}\left(\mathbf{R}_{v}\right)},
$$

where $\operatorname{tr}\left(\mathbf{R}_{v}\right)$ is the sum of the diagonal elements of $\mathbf{R}_{v}$. If the selection of convergence factor is satisfied with Eq. (12), the least mean square algorithm will adjust weights gradually until the filter reaching steady-state mean-square value $\epsilon_{s s}^{W}$. It is defined as

$$
\begin{aligned}
E\left[\epsilon_{s s}^{W}\right] & =\epsilon_{\min }^{W}+\epsilon_{\Delta}^{W} \\
& =\epsilon_{\min }+\epsilon_{\Delta}^{W},
\end{aligned}
$$

where $\epsilon_{\Delta}^{W}$ is the exceeded mean-square value:

$$
\epsilon_{\Delta}^{W}=\frac{1}{2} \mu \operatorname{tr}\left(\mathbf{R}_{v}\right) \epsilon_{\min }^{W} .
$$

It follows that the convergence speed may be controlled by the step size $\mu$. However, decreasing $\mu$ causes a longer convergence time. Therefore, in normalized LMS (NLMS) algorithm [17], the step size is determined, according to an ad-hoc strategy, as a varying parameter that is a function of the power of input signal. That is

$$
\mu=\frac{\alpha}{\sigma_{x}^{2}+\gamma},
$$

where $\alpha, \gamma$ are the positive constants, and $\sigma_{x}^{2}$ is the power of input signal [6]. With accordance to discussion above, the orthogonal signal $\mathbf{v}(k)$ can be obtained from input signal $\mathbf{x}(k)$ which is transformed by Eq. (2). In addition, the adjustment of system weights, $\mathbf{W}$, through the least mean-square in Eq. (11) can also increase convergence speed. It's called the wavelet-based LMS algorithm.

\section{THE PROPOSED STRUCTURE OF ADAPTIVE BEAMFORMING}

Adaptive beamformer is such a spatial filter that combined with sensor array. Spatial sample data which is received by an array in the transmission field, and further adjusted by steering vector $\mathbf{a}_{0}$ of target signal as

$$
\mathbf{a}_{0}=\left[e^{j \phi_{10}}, e^{j \phi_{20}}, e^{j \phi_{30}}, \ldots, e^{j \phi_{M 0}}\right]^{T} .
$$

The parameter $\phi_{i 0}$ signifies the phase difference of target signal between the $i^{\text {th }}$ sensor and the first reference sensor. The steering vector $\mathbf{a}_{0}$ directs at the direction of incident signal in order to dispel other signals which are received by the array in the same phase. Besides, jammer and noise can be suppressed, even can the target signal be received accurately through certain algorithm on the weights adjustment of the beamformer.

Griffiths and Jim [10] exploited unconstrained GSC framework according to the LCMV (linearly constrained minimum variance) proposed by Frost. It decompose the weights vector $w$ into two orthogonal components $\mathbf{w}_{0}$ and $-\mathbf{u}\left(\mathbf{w}=\mathbf{w}_{0}-\mathbf{u}\right)$. Hence, let $\mathbf{w}_{0}$ and $\mathbf{u}$ satisfy the conditions as follow:

$$
\begin{aligned}
& \mathbf{a}_{0}^{T} \mathbf{w}_{0}=f, \\
& \mathbf{a}_{0}^{T} \mathbf{u}=0 .
\end{aligned}
$$

Further, it can be derived as

$$
\mathbf{w}_{0}=\mathbf{a}_{0}^{*}\left[\mathbf{a}_{0}^{T} \mathbf{a}_{0}^{*}\right]^{-1} f .
$$

We consider $\mathbf{u}$ as a product of vector $\mathbf{C}_{j}$ and $\mathbf{w}_{j}(\mathbf{u}=$ $\mathbf{C}_{j} \mathbf{w}_{j}$ ) in which the column vector, $\mathbf{C}_{j}$, appears to be the basis for nullspace of $\mathbf{a}_{0}$, in other words,

$$
\mathbf{a}_{0}^{T} \mathbf{C}_{j}=0 .
$$

Take a traditional GSC framework under consideration which persisting unity gain $(f=1)$ for target signal, as depicted in Fig. 2. We can derive $\mathbf{w}_{0}$ as

$$
\begin{aligned}
& {[1,1, \ldots, 1] \mathbf{w}_{0}=1,} \\
& \Rightarrow \mathbf{w}_{0}=\frac{1}{M}[1,1, \ldots, 1]^{T},
\end{aligned}
$$

where $M$ is an integer. In the upper half plane of Fig. 2, we sum up all sensors output by using weights $\mathbf{w}_{0}$, and take average on it. The weight $\mathbf{w}_{0}$ 


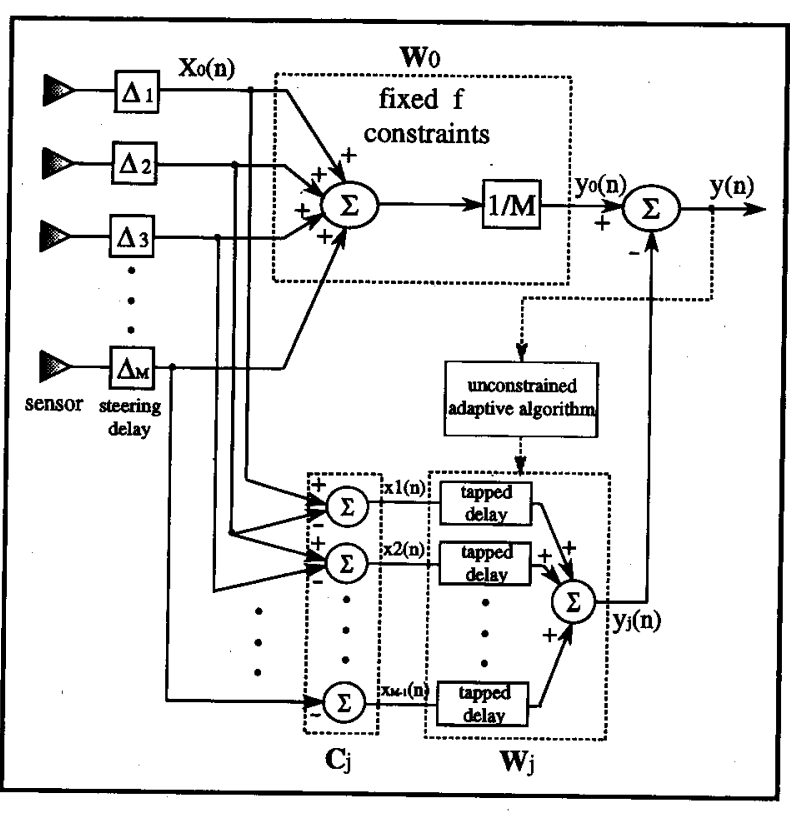

Fig. 2. Block diagram of traditional GSC structure.

is named as fixed target signal in lower half plane. We get

$$
[1,1, \ldots, 1] \mathbf{C}_{j}=\mathbf{0} .
$$

We may select

$$
\mathbf{C}_{j}=\left[\begin{array}{ccccc}
1 & 0 & \cdots & 0 & 0 \\
-1 & 1 & \cdots & 0 & 0 \\
0 & -1 & \cdots & 0 & 0 \\
\vdots & \vdots & \ddots & \vdots & \vdots \\
0 & 0 & \cdots & -1 & 1 \\
0 & 0 & \cdots & 0 & -1
\end{array}\right]
$$

In above equation, $\mathbf{C}_{j}$ is a $M \times(\mathbf{M}-1)$ matrix. Intimately, the actual operation of $\mathbf{C}_{j}$ involves removing the target signal with the same phase. So, $\mathbf{C}_{j}$ is rather known as signal blocking matrix or it may also be considered as reference signal processor in alternative choice. Hence, reference signal $x_{j}, j=1,2, \ldots$, M-1 can be provided by $x_{0}(n)$ after processed by $\mathbf{C}_{j}$. There are (M-1) TDL input signals in the lower plane, and the signal $\mathbf{x}_{i}(n)$ denoted the signal contents of the $i^{\text {th }}$ TDL at time $\mathrm{n}$, it is shown as below:

$$
\mathbf{x}_{i}(n)=\left[x_{i}(n), x_{i}(n-\Delta), \ldots, x_{i}(n-(L-1) \Delta)\right]^{T},
$$

where $\Delta$ is the delay time, and the $\mathbf{w}_{i}$ is the weights vector of the $i^{\text {th }}$ TDL, as depicted as follow:

$$
\mathbf{w}_{i}=\left[w_{1, i}, w_{2, i}, \ldots, w_{L, i}\right]^{T}
$$

Thus, TDL outputs can totally be denoted as

$$
y_{j}(n)=\mathbf{W}_{j}^{T} \mathbf{X}_{j}(n),
$$

where

$$
\begin{aligned}
& \mathbf{X}_{j}(n)=\left[\mathbf{x}_{1}^{T}(n), \mathbf{x}_{2}^{T}(n), \cdots, \mathbf{x}_{M-1}^{T}(n)\right]^{T}, \\
& \mathbf{W}_{j}=\left[\mathbf{w}_{1}^{T}, \mathbf{w}_{2}^{T}, \cdots, \mathbf{w}_{i}^{T}, \cdots, \mathbf{w}_{M-1}^{T}\right]^{T}
\end{aligned}
$$

The quantity $\mathbf{W}_{j}$ is the weight matrix of reference signal produced by lower-half part of Fig. 2. Besides, Fig. 2 reveals that the output signal $y_{0}(n)$ of the fixed signal filter in the upper plane can be expressed as

$$
y_{0}(n)=\mathbf{w}_{0}^{T} \mathbf{x}_{0}(n) .
$$

The $\mathbf{w}_{0}$ can be considered as a fixed target signal filter, while $\mathbf{x}_{0}$ is the output signal processed by steering delay. So, the $y(n)$ output of GSC is obtained from Eqs. (27) and (28) derivations. As a result, we can use the least mean square algorithm of wavelef transform to orthogonalize time-domain signal for weights vector adjustment, by which the processing time of beamformer can be reduced. The framework is illustrated in Fig. 3.

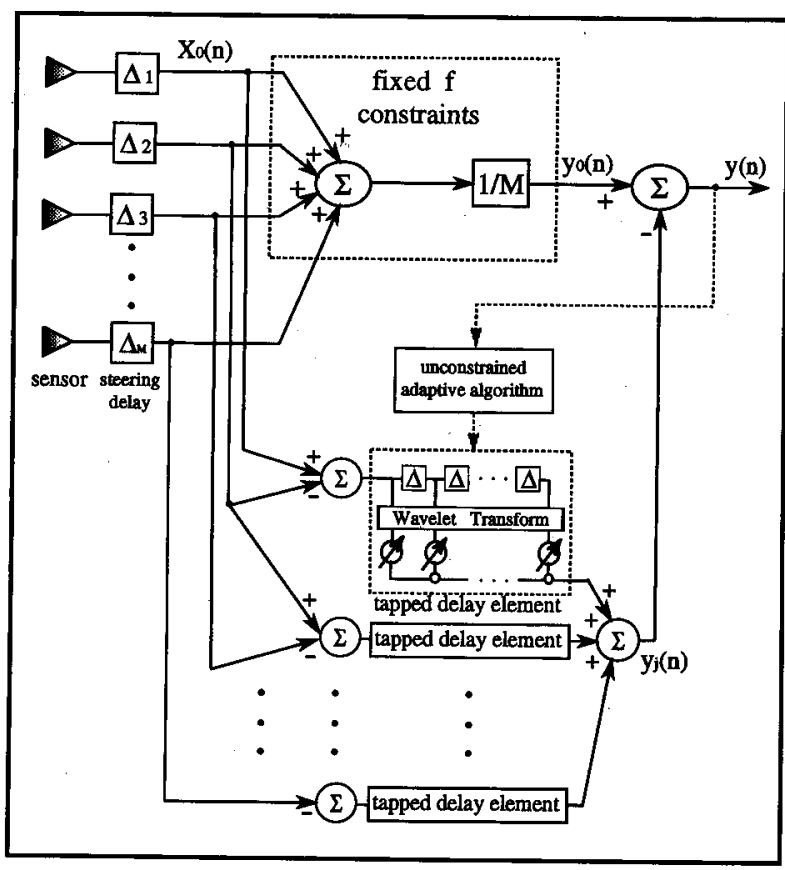

Fig. 3. Block diagram of GSC beamforming with WLMS algorithm. 


\section{EXPERIMENTAL RESULTS}

In all the simulations below, the Haar function is being used as the wavelet basis function for where it may concern. The number of sensors, $M$, is 10 ; while the length of TDL, L, is 17 . The selection of step size must be satisfied with Eq. (12) in order to achieve the convergence.

\section{Case 1:}

Two groups of input signals are used in order to compare the convergence of the least mean-square algorithm in both time and wavelet domains between stationary sinusoidal and nonstationary signals.

1. Sinusoidal signal with frequencies of $150 \mathrm{~Hz}$ and $200 \mathrm{~Hz}$.

2. Time-varying nonstationary signal.

The noise related to both signals is Gaussian white noise with zero mean and 0.04 variance. For constant convergence factor of the least mean-square algorithm, the magnitude of step-size is equal to 0.0005: Fig. 4 is the analysis for stationary signal, while the nonstationary (chirp) signal one is illustrated in Fig. 5. In the case of time-varying convergence factor of the least mean-square algorithm: Fig. 6 is the analysis for stationary signal, while the nonstationary signal one is illustrated in Fig. 7. We discovered in both cases that under identical conditions, the convergence speed of WLMS is verified better than that of TLMS .

Case 2:

In the case of adaptive beamformer, we compare the traditional time-domain LMS algorithm with the wavelet-based LMS algorithm for their effect of beamforming. Assume that beamformer is formed

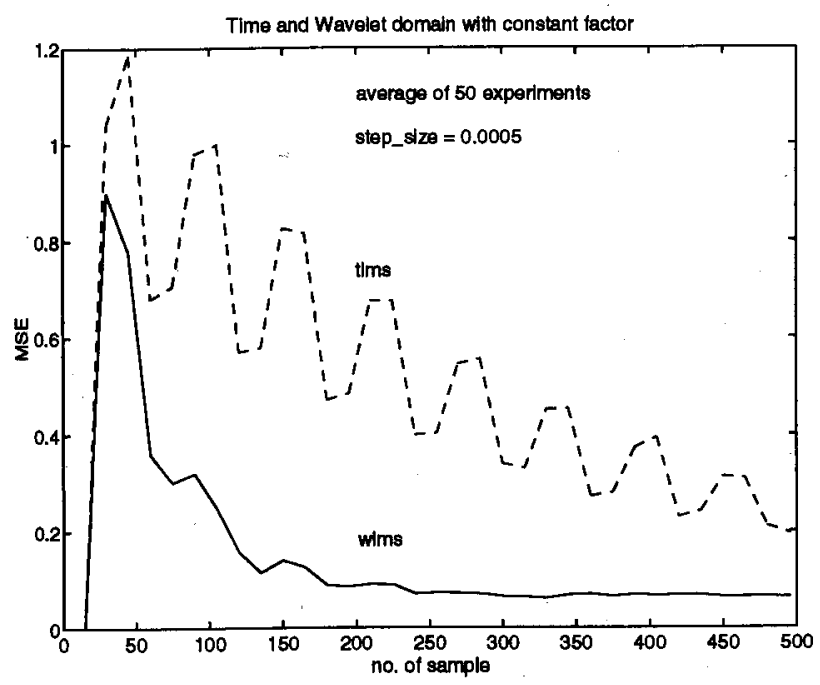

Fig. 4. The comparison between time and wavelet domains learning curves under identical convergence factor for stationary signal (average of 15 samples).

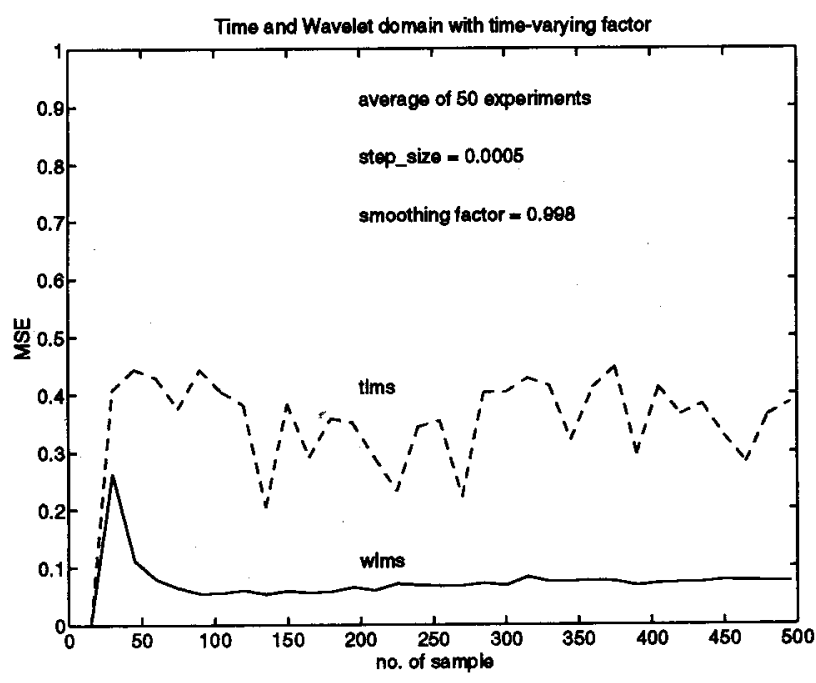

Fig. 5. The comparison between time and wavelet domains learning curves under identical convergence factor for chirp signal (average of 15 samples).

?

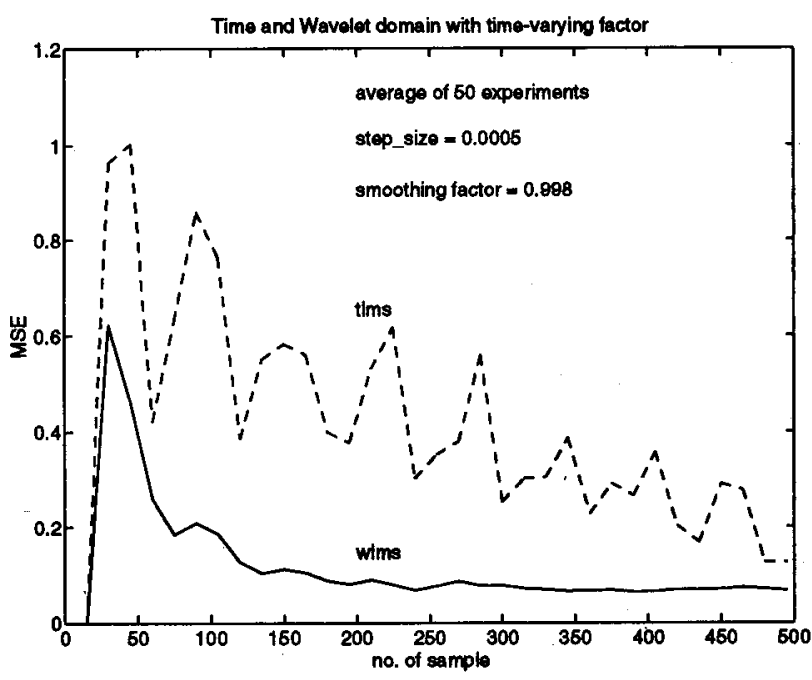

Fig. 6. The comparison between time and wavelet domains learning curves under time-varying convergence factor for stationary signal (average of 15 samples).

by a linear array consisting of 10 sensors, and the signal source to be far-field sinusoidal signal with Gaussian white noise. The sampling time is 1000 and the magnitude of step-size is equal to $8 \times 10^{-6}$. The incident angle for target signal $s(t)$ is $0^{\circ}$ with SNR $=10 \mathrm{~dB}$, where $s(t)=\sin (2 \pi \times 200 \times t)$.

We utilize two groups of jammer, which are stationary and nonstationary respectively with $\mathrm{SNR}=30 \mathrm{~dB}$. For both incident angles of $25^{\circ}$, the $j_{1}(t)$ $=\sin (2 \pi \times 100 \times t)$ and $j_{2}(t)=\sin \left(2 \pi \times\left(-20 t+100 t^{2} / 2\right)\right)$. It revealed in simulation 1 that the learning curve of TLMS-GSC converges slowly under identical con- 


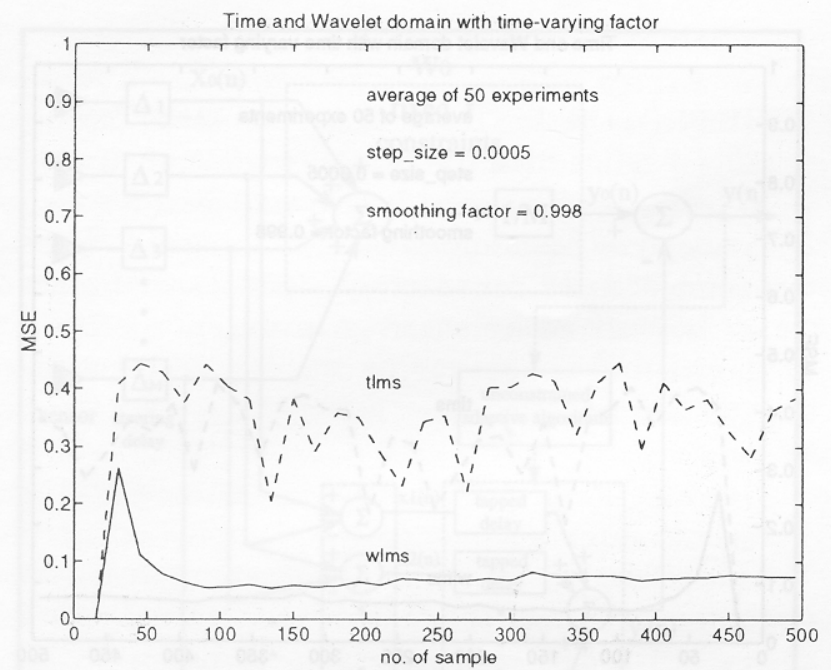

Fig. 7. The comparison between time and wavelet domains learning curves under time-varying convergence factor for chirp signal (average of 15 samples).

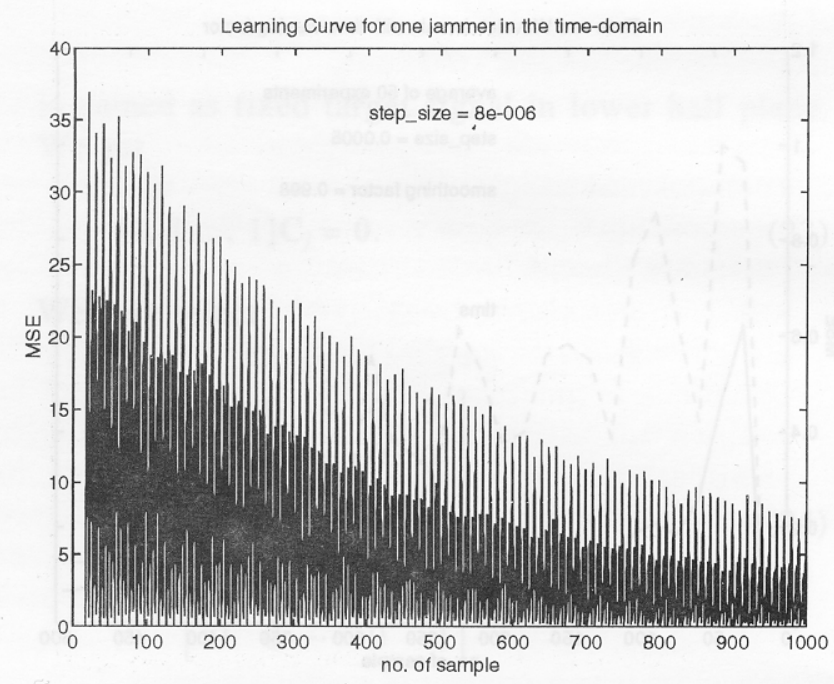

Fig. 8. Learning curve of the application of TLMS-GSC on a sinusoidal jammer.

ditions and it's depicted in Fig. 8. Eventually, it caused even can the jammer coming from $25^{\circ}$ not able to be suppressed successfully as shown in Fig. 10. On the other hand, the learning curve converges rapidly to steady-state by using WLMS-GSC framework, as given in Fig. 9, furthermore, it also suppresses the jammer come from $25^{\circ}$ as in Fig. 11. But under the identical conditions for simulation 2 , the learning curve of TLMS-GSC converges slowly as depicted in Fig. 12, as a result, it even unable to suppress jammer successfully coming from $25^{\circ}$ as shown in Fig. 14. In contrast, it would arrive at a

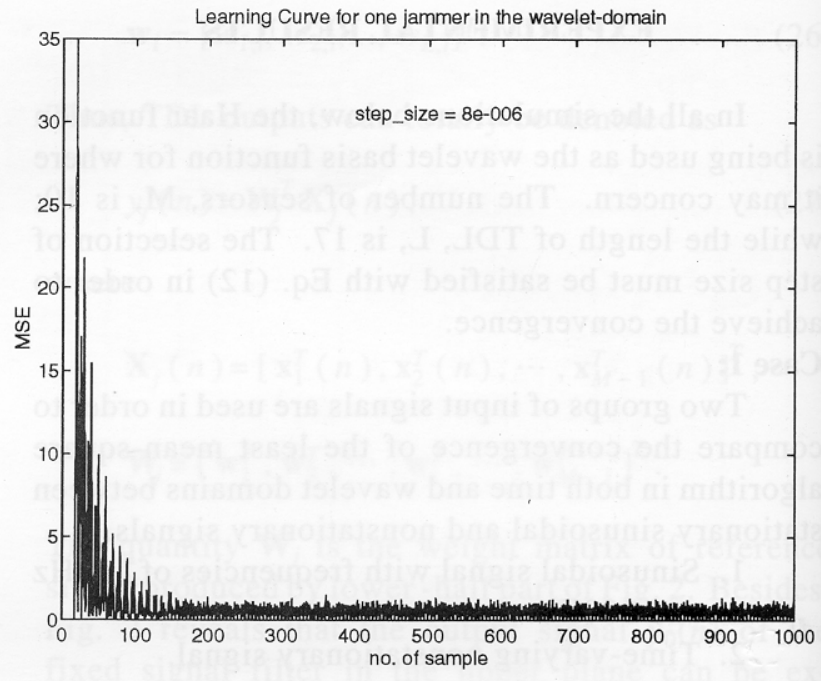

Fig. 9. Learning curve of the application of WLMS-GSC on a sinusoidal jammer.

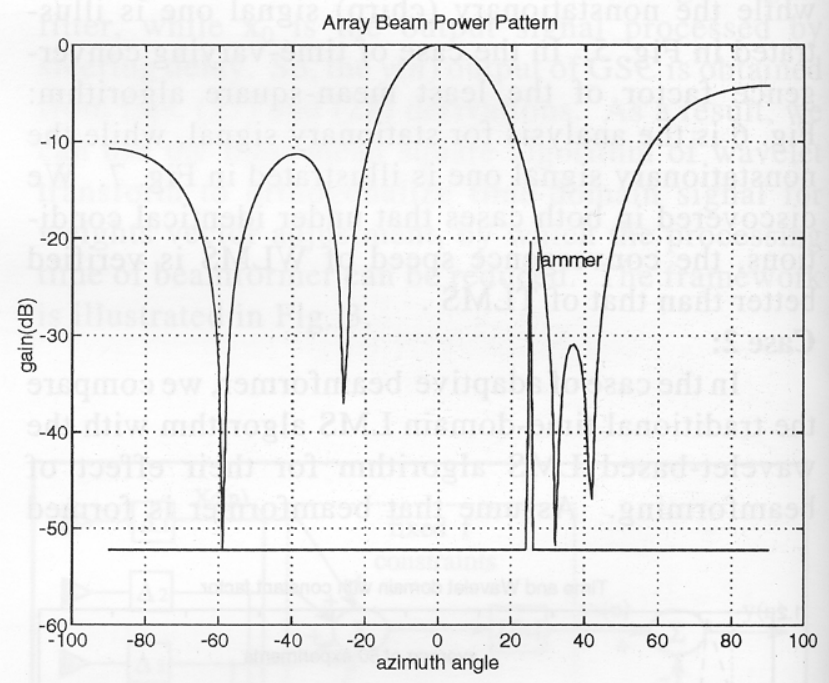

Fig. 10. Beampattern of TLMS-GSC algorithm for stationary signal.

steady-state with the usage of WLMS-GSC framework, as given in Fig. 13. In addition, suppression of jammer coming from $25^{\circ}$ is achieved as illustrated in Fig. 15. In supplementary, the simulation upon jammer coming from $-20^{\circ}$ also indicates that our proposed WLMS-GSC performing suppression successfully as in Fig. 16 than the suppression of TLMSGSC in Fig. 17.

\section{CONCLUSION}

In this paper, a brand new tool of signal analysis 


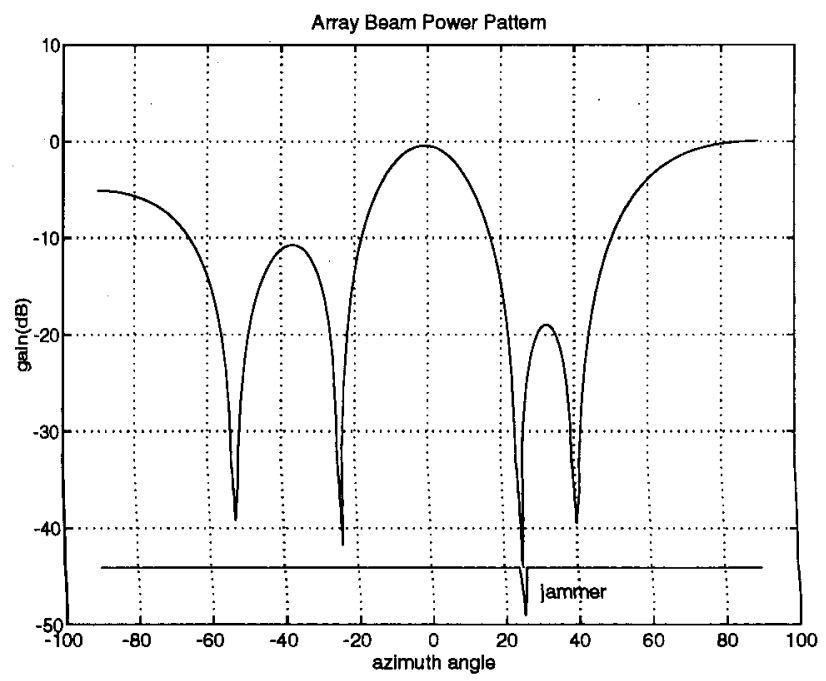

Fig. 11. Beampattern of WLMS-GSC algorithm for stationary signal.

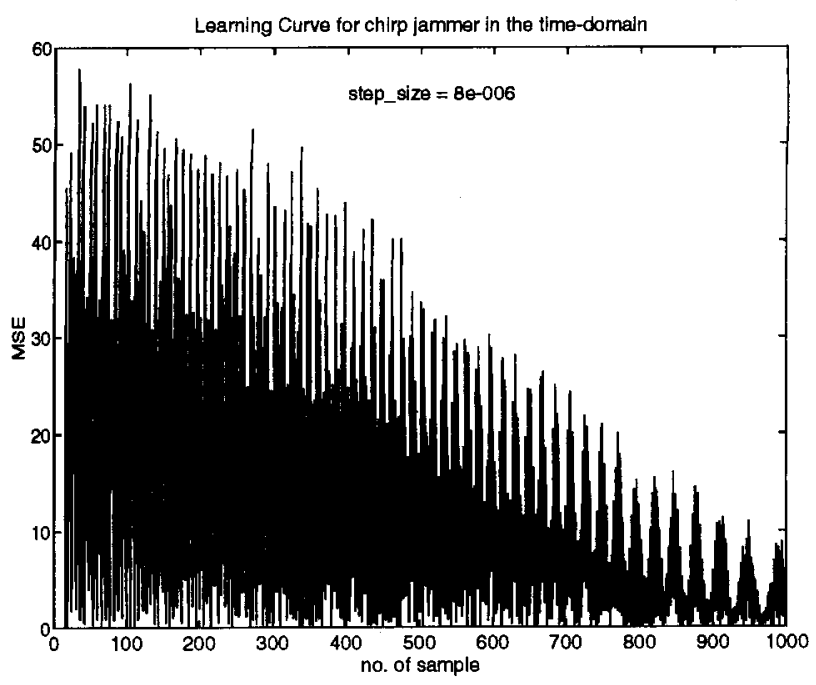

Fig. 12. Learning curve of TLMS-GSC for nonstationary signal.

wavelet transform in which maintaining good resolution in time-frequency plane is discussed. Further, the multiresolution feature of wavelet transform is being introduced in order to improve the convergence of the least mean-square algorithm. And it has been proven better if compare with that of traditional time-domain. The least mean-square algorithm in wavelet domain can force weights to adjust rapidly no matter for stationary sinusoidal signal or nonstationary chirp signal. These are shown in simulation case 1 . Hence, the application of the mean-square algorithm on GSC frameworked beamformer, as depicted in simulation case 2 , can effectively suppress stationary or nonstationary jammer. In addition, it makes the target signal received by array more

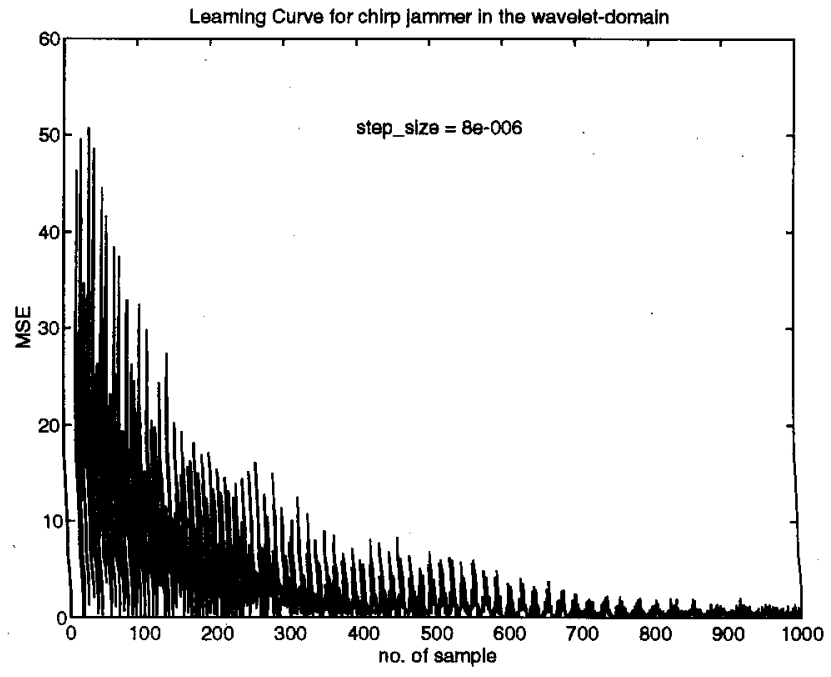

Fig. 13. Learning curve of WLMS-GSC for nonstationary signal.

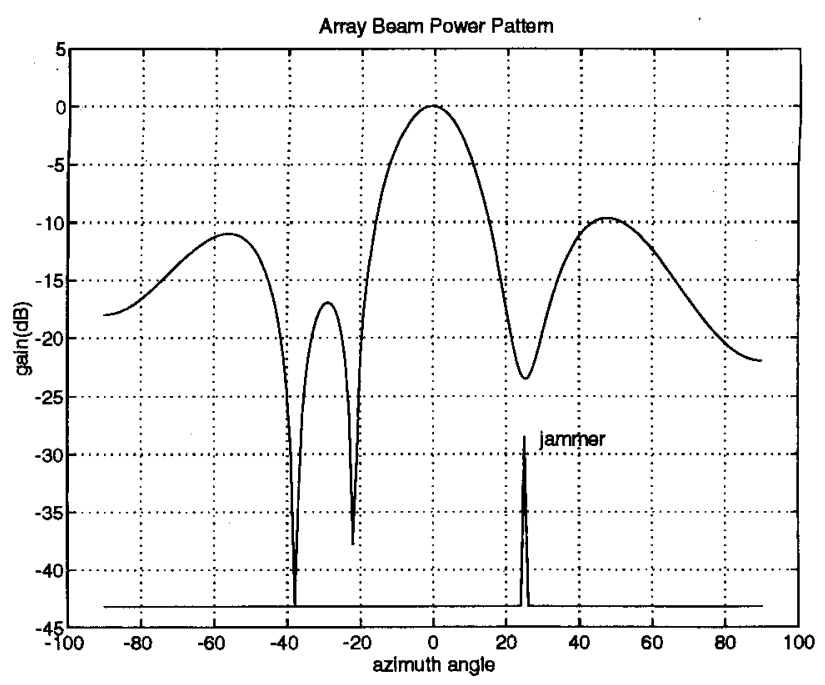

Fig. 14. Beampattern of TLMS-GSC algorithm for nonstationary signal.

efficient and in real-time manner.

\section{ACKNOWLEDGEMENTS}

This work was supported by National Science Council, Taiwan, R.O.C. under the program NSC 842213-E-019-015. The authors also wish to thank the reviewers for numerous helpful suggestions.

\section{REFERENCES}

1. Haykin, S., Adaptive Filter Theory, 3rd edition, Prentice-Hall, New Jersey (1996).

2. Clarkson, Peter M., Optimal and Adaptive Signal Processing, CRC Press (1993). 


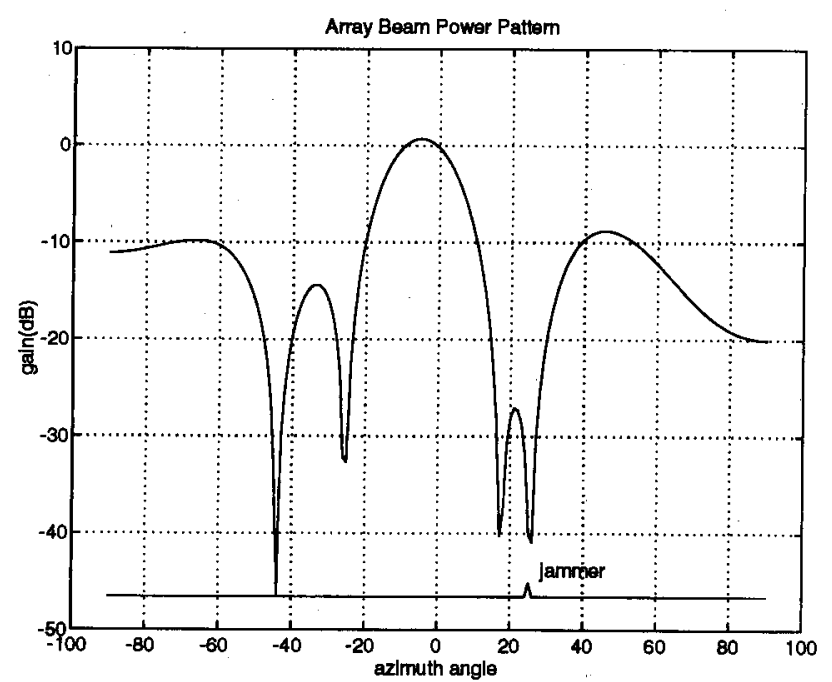

Fig. 15. Beampattern of WLMS-GSC algorithm for nonstationary signal.

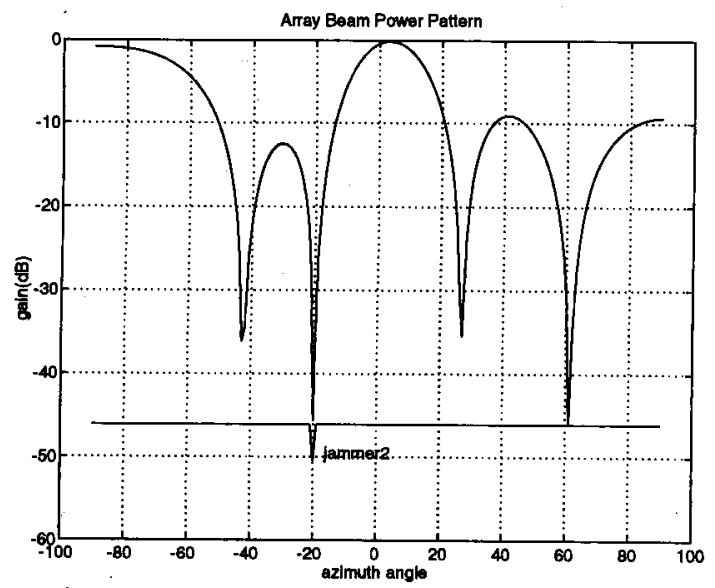

Fig. 16. Beampattern of WLMS-GSC algorithm for jammer coming from $-20^{\circ}$.

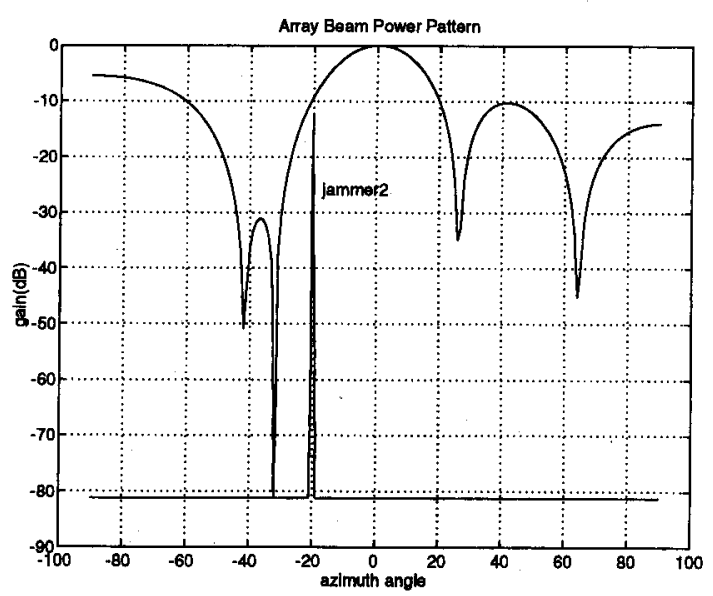

Fig. 17. Beampattern of TLMS-GSC algorithm for jammer coming from $-20^{\circ}$.
3. Van Veen, B.D. and Buckley, K.M.,"Beamforming: A Versatile Approach to Spatial Filtering," IEEE ASSP Magazine, April, pp. 4-24 (1988).

4. Jouny, I., "Applications of Wavelet Subband Decomposition in Adaptive Arrays," 1994 International Symposium IEEE Antennas and Propagation, pp. 1619 (1994).

5. Jouny, I., and Culpepper, "Adaptive Hot Clutter Mitigation Using Subbanding," Proceedings of the IEEE-SP International Symposiumon Time-Frequency and Time-Scale Analysis, pp. 476-479 (1994).

6. Lee, J.C., and Un, C.K., "Performance of Transformdomain LMS Adaptive Digital Filters," IEEE Transactions on Acoustics, Speech, and Signal Processing, Vol. ASSP-34, pp. 499-510 (1986).

7. Erdol, N., and Basbug, F., "Performance of Wavelet Transform Based Adaptive Filters," Proc. ICASSP 1993, vol. III, pp. 500-503 (1993).

8. Erdol, N., and Basbug, F., "Wavelet Transform Based Adaptive Filters: Analysis and New Results," IEEE Transactions on Signal Processing, Vol. 44, No. 9, pp. 2163-2171 (1996).

9. Daubechies, I., "Orthonormal Bases of Compactly Supported Wavelets," Communications on Pure and Applied Mathematics, Vol. XLI, pp. 909-996 (1988).

10. Griffiths, L.J., and Jim, C.W., "Alternative Approach to Linear Constrained Adaptive Beamforming," IEEE Transactions Antenna Propagation, Vol. AP-30, pp. 469-478 (1982).

11. Frost, O.L. III, "An Algorithm for Linearly Constrained Adaptive Array Processing," Proceeding IEEE, Vol. 60, No. 8, pp. 926-935, Aug. (1972).

12. Goldstein, J.S. and Ingram, M.A., "Reduced Complexity Adaptive Structures for Jam-Resistant Satellite Communications," IEEE MILCOM'93, Vol. 3, pp. 1033-1037 (1993).

13. Srinath H. and Tewfik, A.H., "Wavelet Transform Domain LMS Algorithm," Proc. ICASSP 1993, Vol. III, pp. 508-510 (1993).

14. Wiener, N., Extrapolation, Interpolation and Smoothing of Stationary Time Series, with Engineering Applications, Wiley, New-York (1949).

15. Kailath, T., "A View of Three Decades of Linear Filtering Theory," IEEE Transactions Information Theory, Vol. IT-20, pp. 145-181, Mar. (1974).

16. Doroslovački, M. and Fan, H., "Wavelet-Based Adaptive Filtering," Proc. ICASSP 1993, Vol. III, pp. 488-491 (1993).

17. Bitmead, R.R., and Anderson, B.D.O., "Performance of Adaptive Estimation Algorithms in Dependent Random Environments," IEEE Trans. Automatic Control, Vol. AC-25, pp. $788-794$ (1980). 
指波域最小均方演算法應用於可適 性波束構成器之研究

張 順 雄 張 晋 彰

國立台灣海洋大悬電機工程學系

摘 要

指波轉换為一種利用原型指波基底函數的撗 張及收缩, 而在時刻度平面具彈性視窗的轉换演算 法。因其對於訊號之高、低頻率成份具有良好的解 析效果, 故我們將其應用於停统的最小均方演算法 (LMS)。時域的輸入訊號經轉换後將於指波域具有 正交性, 此外, 可籍此提界LMS演算法學習曲線之 收敛速度。我們稱此方法為指波域最小均方演算法 (WLMS)。在本論文我們將WLMS應用於傮統的可 通性波束構成器中, 藉此取代時域最小均方演算法 (TLMS) 以調整系統之權重, 並提界可適性波束構 成器的收敛速度, 此外, 我們將驗証其理碖並且以 電腦模擬所提方法之放能。 